\title{
Nuevos modelos de negocios periodisticos. El caso de SUR ON+ y su incidencia en Twitter
}

New journalistic business models. The case of SUR ON+ and its impact on twitter

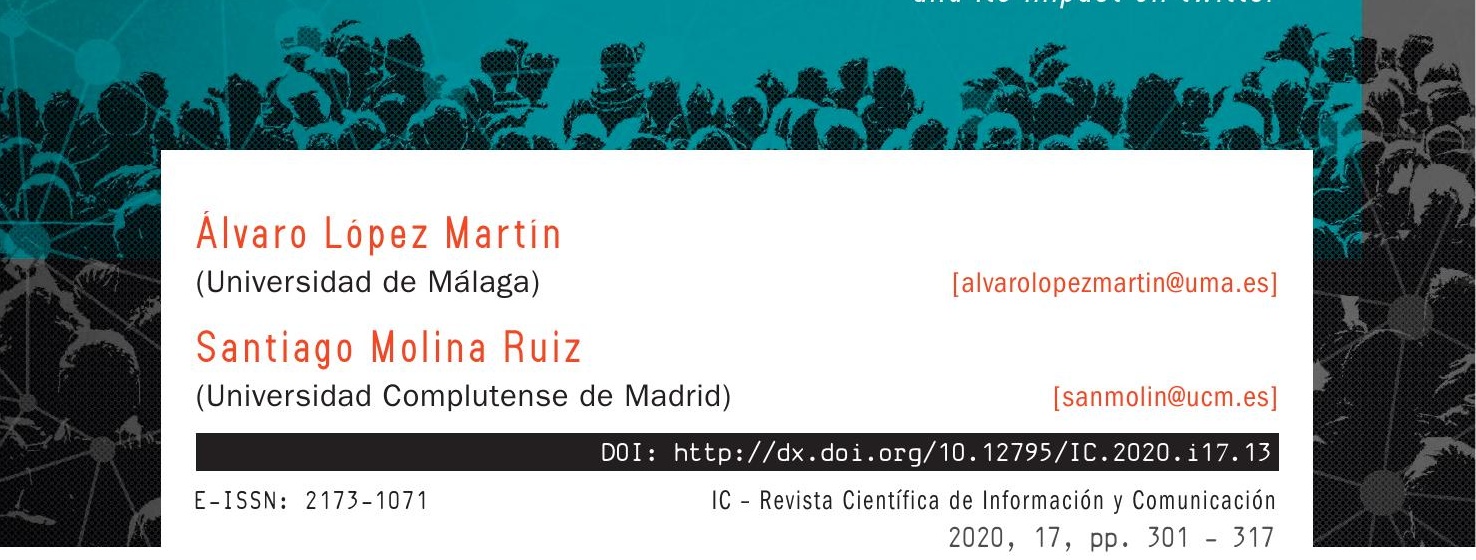

2020, 17, pp. $301-317$

\section{Resumen}

En los últimos años diversas cabeceras están comenzando a implantar muros de pagos. Una de las últimas en sumarse a ello ha sido Sur. El análisis de los tuits referidos a SUR ON+ revela que este asunto tuvo una incidencia moderada entre los temas abordados. Aun así, la mayoría de los usuarios se muestran críticos con este modelo de negocio y se resisten a pagar por contenidos periodísticos.

\section{Abstract}

In the last years various newspapers are beginning to implement paywalls. One of the last to join it has been Sur. The analysis of tweets referring to SUR ON+ reveals that this issue had a moderate incidence among the topics addressed. Most users are critical of this business model and are reluctant to pay for journalistic contents.

Palabras clave

Periodismo, prensa local, muro de pago, modelo de negocio, Twitter.

\section{Keywords}

Journalism, local press, paywall, business model, Twitter. 


\section{Sumario}

1. Introducción

2. Estado de la cuestión

2.1. La influencia de internet en el nuevo ecosistema periodístico

2.2. Elmuro de pago: en busca de un modelo de negocio sostenible

3. Objetivos

4. Metodología

5. Resultados

5.1. Incidencia

5.2. Reacciones sobre el muro de pago

6. Conclusiones

7. Bibliografía

\section{Summary}

1. Introduction

2. State of the matter

2.1. The influence of the internet on the new journalistic ecosystem

2.2. The paywall: in search of a sustainable business model

3. Objectives

4. Methodology

5. Results

5.1. Incidence

5.2. Reactions about paywall

6. Conclusions

7. Bibliography 


\section{Introducción}

En los últimos años las empresas periodísticas han iniciado un camino de cambios y transformación de sus respectivos modelos de negocio con el propósito de poner fin, al menos de manera parcial, a la cultura del "todo gratis" que se generalizó con el surgimiento de las ediciones digitales, a través de las cuales los lectores podían acceder a los contenidos del periódico gratuitamente. De esta forma, los medios de comunicación aspiran a desarrollar un modelo de financiación que siga haciendo del periodismo y/o la información un negocio rentable. Un propósito al que se han visto abocadas estas empresas a raíz de la crisis económica mundial y del sector de la comunicación, así como de la irrupción de las nuevas tecnologías, dos factores que han motivado, en gran medida, un "colapso del modelo de negocio tradicional" (Palau-Sampio, 2016:64). Hasta ahora, la principal fuente de ingresos en la web procedía de la publicidad display, algo que "no solo es insuficiente para rentabilizar los medios, sino que esa partida está decreciendo" (Gómez-Borrero, 2014: 1). Las grandes inversiones publicitarias se destinan actualmente a los buscadores más importantes, como Google y las redes sociales como Facebook 0 Twitter, en detrimento de los medios tradicionales.

Ante este cambiante ecosistema mediático, diversas cabeceras han puesto en marcha distintos modelos de pago o suscripción, conocido como paywall o muro de pago. Si bien este modelo presenta varias modalidades o tipologías - muro 'duro', aquel en el que hay que pagar para acceder al contenido; o muro 'blando', se permite el acceso gratuito a un número de noticias determinadas cada mes-,medios internacionales de referencia ya han aplicado esta medida de una u otra manera, como The New York Times, The Daily Telegraph, The Washington Post, Le Monde, The Times, Folha do Sao Paolo, Die Welt o Helsingin Sanomat. Sin embargo, en España las principales cabeceras se resisten a implantar de manera decidida este modelo de negocio, algo que sí está llevando a cabo el grupo Vocento paulatinamente con sus diarios regionales, un camino iniciado por El Correo en el año 2015. Uno de los últimos diarios en los que Vocento ha puesto en marcha el muro de pago es Sur, periódico líder de Málaga con más de 80 años de tradición en la provincia. El paywall de este diario malagueño, aplicado bajo la denominación de SUR $0 N+$, fija el límite de visitas gratuitas mensuales en cinco noticias, siendo el coste inicial de la suscripción de 4,95 euros por mes. De acuerdo a la clasificación de GómezBorrero (2014), el modelo implantado por la cabecera de Vocento es de carácter freemium, dado que, además, de existir un límite de visitas gratuitas, también cuenta con contenido exclusivo para los suscriptores. 


\section{Estado de la cuestión}

\subsection{La influencia de internet en el nuevo ecosistema periodístico}

A lo largo de la historia los avances tecnológicos han condicionado y ejercido una notable influencia en el desarrollo de la profesión periodística. Especialmente en las dos últimas décadas, la irrupción de las ediciones digitales y las redes sociales, así como el auge de todo aquello relacionado con internet, han sacudido los cimientos de la industria periodística, motivando el surgimiento de un nuevo ecosistema o contexto comunicativo en el que muchos medios de comunicación han adoptado la mentalidad digital first. Esta línea de actuación consiste en "cambiar las inercias, dejando de pensar en la edición en papel como prioridad y situando lo digital en el centro de su negocio y de sus servicios" (Guallar, 2015: 154).

Algunos autores como Peñafiel (2016) exponen una visión maximalista y considera que estas innovaciones han supuesto un cambio del paradigma de la comunicación. Cabrera abunda en este asunto y señala que, para los medios, "la presencia en internet no es una recomendación, sino una obligación" (2016: 43), algo que ya vaticinaba Salaverría, quien afirmaba que "entre los medios grandes y pequeños contar con una edición electrónica se había convertido ya en un asunto de competencia y prestigio" (2005: 10).

No obstante, Picard (2014) incide en algunas de las tendencias asociadas al notable protagonismo de la web: el debilitamiento de las empresas periodísticas, el amplio abanico de medios o la fragmentación de las audiencias, entre otras. En esta misma línea, Rodríguez y Favaretto señalan que este nuevo paradigma comunicacional ha agravado la crisis que afronta la profesión y "son muchos, medios y periodistas, los que se han visto incapacitados para adaptarse al nuevo entorno y aceptar la reforma transversal que la red ha incitado en el modelo de negocio" (2017: 211). Al respecto, Aguado y Castellet apuntan que "la crisis de la industria periodística viene a coincidir con un estallido de la demanda de contenido y la diversidad de ámbitos y formas de consumo informativo" (2013: 199).

Otro de los elementos que desempeñan un importante papel en este nuevo ecosistema son las redes sociales, las cuales "amplían exponencialmente en el nuevo siglo el ecosistema mediático" (Campos-Freire et al., 2017: 43). Diversos autores (Campos-Freire et al., 2016; Justel-Vázquez et al., 2018) ponen en valor la importancia de las redes sociales "como instrumentos que conservan y proyecta el valor del medio más allá de sus negocios tradicionales (Campos-Freire et al., 2016: 454). Asimismo, la notable incidencia de estas 
nuevas vías sobre los medios de comunicación se ve reforzada en el estudio de García-Avilés y Arias (2016), quienes hablan del surgimiento de géneros periodísticos exclusivos para las redes sociales. La figura del periodista también se ha visto transformada a raíz del desarrollo de las ediciones digitales y las redes sociales. Así, el perfil de profesional de la información que en la actualidad contratan desde las empresas informativas está definido en torno a las competencias digitales (Palomo y Palau-Sampio, 2016).

Si bien las redes se posicionan como una de las principales vías para acceder y comentar noticias -como se recoge en El Libro Blanco de la Información 2017, el 56,8\% de los lectores de prensa en España empleaban las redes sociales para consultar las noticias-, sirven también como importantes canales de expresión de las audiencias, que dejan de ser pasivas y se convierten en prosumidoras, disponiendo de "autonomía para producir, distribuir y consumir los contenidos que circulan por las redes" (De Aguilera y Casero-Ripollés, 2018: 7). Vinculado con esto, Guallar (2015) sostiene que en la actualidad las audiencias demandan a los medios de comunicación interactividad. En términos similares se expresa García Galindo, quien expone que "la opinión pública actual no se construye solo a partir de lo que dicen los medios, sino a través de la interacción con los medios" (2010: 275), una acción para la que internet -y especialmente las redes sociales- es la principal vía para lograr ese propósito. No obstante, Guallar incide que "la prensa digital no parece estar resolviendo del todo bien esta exigencia", a lo que añade que "Ios espacios de participación que ofrece suelen estar por debajo de las expectativas" (2015: 155).

\subsection{El muro de pago: en busca de un modelo de negocio sostenible}

El nacimiento de las ediciones digitales trajo consigo un aumento considerable de las audiencias, las cuales tenían acceso de manera gratuita a los mismos contenidos que los medios vendían en sus ediciones impresas. Esta decisión de los editores de prensa imposibilitó aprovechar una gran oportunidad para monetizar y obtener rendimiento económico de esos nuevos lectores. Esto, como señala Ormaetxea, contribuyó en gran medida "a extender la cultura de la gratuidad digital" (2014: 55).

Actualmente, una de las principales preocupaciones de la profesión periodística "es afrontar la dominante condición del ‘todo gratis' que internet ha impuesto" (Rodríguez \& Favaretto, 2017: 212). En los últimos años tres factores han condicionado el sector de la prensa a nivel mundial: la caída de las ventas de ejemplares, la reducción de ingresos 
por publicidad y la disminución de las plantillas (Palau-Sampio, 2016). En palabras de la citada Palau-Sampio, "entre 2007 y 2013 los ingresos por publicidad se han reducido un 65\% en la prensa diaria” (2016: 64). Esta caída continúa a un ritmo notable y durante el primer semestre de 2019 la inversión publicitaria dirigida a los diarios disminuyó un 7,8\% respecto al mismo periodo del año 2018 (Infoadex, 2019). Especialmente los dos primeros factores mencionados anteriormente han motivado que los medios de comunicación busquen otras alternativas para seguir haciendo del periodismo un negocio rentable y sostenible, después de que el modelo de negocio tradicional -venta de ejemplares y de espacio publicitario- genere más dudas e incertidumbres que nunca.

Este contexto ha derivado en que muchos medios impongan un nuevo modelo de negocio basado en el pago por los contenidos periodísticos - por la totalidad o algunos de ellos- de las ediciones digitales, lo cual se conoce como "muro de pago" o paywall. A su vez, se pueden distinguir distintas categorías o modelos relacionados con esta medida, ya aplicada y con un largo recorrido en diarios estadounidenses y de Europa del norte y Australia, donde la media de ingresos del paywall respecto al total "alcanza el 20 por ciento" (Campos-Freire et al., 2017: 45). Sin embargo, no hay que perder de vista que, como incide Palau-Sampio, "la disyuntiva entre gratuidad y pago está lejos de quedar resulta" (2016: 64-65), por lo que tanto desde los medios pequeños hasta los diarios de referencia se aprecia "un esfuerzo conjunto para encontrar fórmulas de negocio que permitan la aceptación de los muros de pago" (Rodríguez y Favaretto, 2017: 217). Aun así, la aplicación del muro de pago "no está siendo todo lo mayoritaria que se suponía" (Guallar, 2015: 154).

Distintos autores han distinguido variantes y modelos relacionados con el paywall (Meso et al., 2014; Campos-Freire et al., 2017; González Alba, 2018), aunque es Ormaetxea quien realiza una clasificación más clarividente, diferenciando entre muro de pago "duro" y "suave" (2014: 55). En el primer caso, se "requiere suscripción para tener acceso a la mayor parte o todo el contenido digital de calidad", mientras que con el segundo modelo de negocio se permite el acceso a "un número determinado de artículos gratis cada mes antes de requerir una suscripción" (Ormaetxea, 2014: 55-56). A estas tipologías habría que añadir una tercera, un muro de pago "híbrido" - o conocido también como freemium (Gómez-Borrero, 2014)-, el cual se correspondería con el modelo de negocio implantado por el grupo Vocento en sus periódicos regionales, con límite de visitas mensuales y, además, contenidos exclusivos para sus suscriptores.

En relación a la disposición de los lectores por pagar por los contenidos periodísticos, un estudio de Rodríguez y Favaretto (2017) recoge que una gran parte de 
la audiencia no está dispuesta a pagar por información mientras pueda satisfacer sus necesidades informativas en otros medios, a lo que añaden que la percepción predominante en España es solo aquellas personas con intereses específicos pagarán por contenidos periodísticos. En relación a esto, Goyanes (citado en Palau-Sampio, 2016: 64)añade que los internautas presentan una mayor disposición a pagar por aquellos contenidos "para dispositivos móviles o vinculados a servicios complementarios".

Frente a esto, Ormaetxea cuestiona este modelo de negocio y considera que "ya hay suficiente experiencia para saber que esta barrera tecnológica no será la solución a los problemas de la prensa" (2014: 55). Al respecto, Gómez-Borrero señala que "encontramos medios que están deshaciendo el camino y diarios como San Francisco Chronicle y The Dallas Morning News han tenido que derribar sus muros de pago por no obtener los resultados esperados" (2014: 2). Sin embargo, esto puede estar siendo fruto de una incuestionable cultura del "todo gratis" implantada lustros atrás, la cual junto a la aparición de las redes sociales y otras circunstancias han motivado una devaluación de la información, siendo en la actualidad una difícil tarea y uno de los grandes retos de la industria de los medios monetizar los contenidos periodísticos. Este hecho ocurrirá, según Rodríguez y Favaretto, "cuando la audiencia logre entender la diferencia entre la comunicación libre, el valor añadido y la información diaria convencional” (2017: 218).

\section{Objetivos}

La presente investigación tiene como objetivo fundamental conocer la incidencia y las reacciones que generó en Twitter la implantación del muro de pago (SUR ON+) del diario malagueño Sur, perteneciente al grupo Vocento, lo que, a su vez, nos permitirá dilucidar parcialmente el grado de aceptación o rechazo que provocó este nuevo modelo de negocio entre sus lectores.

Asimismo, como vía de concreción del propósito general de la investigación se han establecido tres objetivos subsidiarios. El primero de ellos es explorar en qué medida se registran tuits referidos al muro de pago respecto al total, lo quepermitirá también determinar la incidencia que tuvodicho asunto en esta red social (01). Relacionado con el propósito anterior está el de conocer las temáticas principales y cuestiones más destacadas que se han abordado a la hora de hablar sobre el muro de pago, identificando, además, si los mensajes son de carácter positivo (aceptación) 0 negativo (rechazo) (02). Por último, se pretende determinar si, a raíz del nuevo sistema 
de pago, los internautas manifiestan en sus tuits una intención o voluntad de seguir siendo lectores habituales de ese medio o si, por el contrario, indican que dejarán de leer dicha cabecera (03).

\section{Metodología}

El análisis de contenido se presenta como el método más apropiado para alcanzar los objetivos de la investigación planteados. La elección de esta técnica responde a su idoneidad para el estudio de un volumen elevado de información -en este caso, los tuits registrados-, así como su versatilidad instrumental. Así se desprende de lo recogido por Barredo, quien destaca que la flexibilidad de esta técnica posibilita "adaptarse a fenómenos muy diversos y a ámbitos simbólicos distantes” (2015: 27).

Por su parte, Neuendorf considera que esta técnica "no se limita en cuanto a los tipos de variables que pueden medirse o el contexto en el cual los mensajes son creados o presentados" (2002: 9). Este mismo autor defiende que el análisis de contenido goza de "ciertas características adicionales que lo colocan en una posición única como la metodología principal para el estudio de mensajes" (Neuendorf, 2002: 9). En relación a esto, este método "permite examinar científicamente tanto los significados como los significantes de cualquier texto" (Igartua, 2006: 181).

Asimismo, cabe mencionar las tres características principales que Wimmer y Dominick (1996) asocian a esta técnica: sistematicidad, objetividad y cuantificación. Una serie de rasgos que favorecen que las "descripciones o explicaciones de los fenómenos [...] no estén sesgadas por las particularidades del investigador" (Igartua, 2006: 182). En esencia, se podría decir, según Igartua, que el análisis de contenido "permite descubrir el ADN de los mensajes mediáticos" (2006: 181).

En cuanto a la muestra, en esta investigación se pone el foco sobre las reacciones de los internautas en Twitter relativas a la aplicación SUR ON+, es decir, el muro de pago de la cabecera malagueña Sur. Para ello, se han analizado todos los tuits $(n=488)$ publicados entre el 7 y el 20 de mayo de 2019 en los que los usuarios mencionan o interactúan con el perfil oficial del medio (@DiarioSUR) en esta red social.

La elección de dicho periodo (del 7 al 20 de mayo de 2019) se debe a que este intervalo corresponde a la semana anterior y posterior a la fecha en la que comenzó a aplicarse el límite de visitas gratuitas en la página web de Sur, el martes 14 de mayo de 2019. De esta forma, se podrá conocer la incidencia de este asunto, por un lado, 
durante los días previos -en los cuales ya había sido anunciada esta medida- y, por otro, una vez implantada.

Aunque son varios los diarios españoles que ya han comenzado a aplicar el límite de visitas en sus ediciones digitales, la elección del caso de Sur para la muestra se debe a que es una de las últimas cabeceras de Vocento -desde 2015 este grupo está aplicando de manera paulatina esta medida a sus diarios regionales- en adoptar este modelo de negocio. Asimismo, este diario generalista es el principal referente de la prensa malagueña, posicionándose en cabeza en cuanto a número de lectores -según la segunda oleada de 2019 del Estudio General de Medios (EGM) esta cabecera cuenta con 123.000 lectores- respecto al resto de diarios locales de Málaga.

Para la ejecución de la investigación y análisis de las piezas se ha diseñado la siguiente ficha de análisis, la cual está constituida por dos apartados:

a) Información de registro: Aquellos datos relativos a la identificación del tuit.

b) Análisis de contenido: En este bloque se contemplan diversas variables con las que se pretende conocer la incidencia y opinión generada relativa al paywall:

- Temática: En este apartado se pretende identificar cuántos tuits se refieren a nuestro objeto de estudio, lo que permitirá conocer también la incidencia que tiene respecto al total de los mensajes registrados.

- Motivo aceptación/rechazo: Relacionado con los mensajes relativos al muro de pago, se explorará en qué medida los usuarios manifiestan una opinión favorable o negativa a este modelo de pago. Asimismo, se pondrá el foco en los argumentos aportados por estos en sus tuits, diferenciando, entre las principales causas que motivan su aceptación o rechazo la publicidad, la calidad, el precio, el propio modelo de negocio y/o el mínimo de lecturas gratuitas fijadas.

- Futuro: Se analizará si los usuarios muestran en los tuits registrados su intención o voluntad acerca de seguir leyendo dicha cabecera una vez que se aplique el límite de visitas. 


\section{Resultados}

\subsection{Incidencia}

Los resultados del análisis de contenido permitieron constatar que esta cuestión tiene una moderada incidencia respecto al total de mensajes o tuits registrados. Concretamente, a lo largo de las dos semanas objeto de estudio se contabilizan un total de 488 tuits dirigidos a la cabecera de Vocento, solo refiriéndose 53 de ellos (10,86\%) al muro de pago.

Pese a que la puesta en marcha de esta medida se anunció desde varias semanas antes a la fecha fijada para el comienzo del cobro por contenidos, durante los días previos al 14 de mayo este asunto fue escasamente abordado por los usuarios en Twitter, solo registrándose 13 mensajes referidos al objeto de estudio de los 234 tuits totales, lo que supone solo un 5,56\%. A partir de su aplicación, el interés o atención por el muro de pago aumenta, lo que se manifiesta en una multiplicación de los tuits referidos a SUR ON+. En este sentido, en la semana del 14 al 20 se contabilizan un total de 254 tuits en los que se mencionan a @DiarioSuR, de los cuales $40(15,75 \%)$ tratan sobre el muro de pago. Esta cifra supone, además, un incremento de un $207,69 \%$ de tuits sobre este asunto respecto a la semana anterior, previa a su puesta en marcha.

\section{FIGURA 1}

Incidencia del muro de pago respecto al total de tuits dirigidos a @DiarioSUR

SEMANA 2

(DEL 14 AL 20)

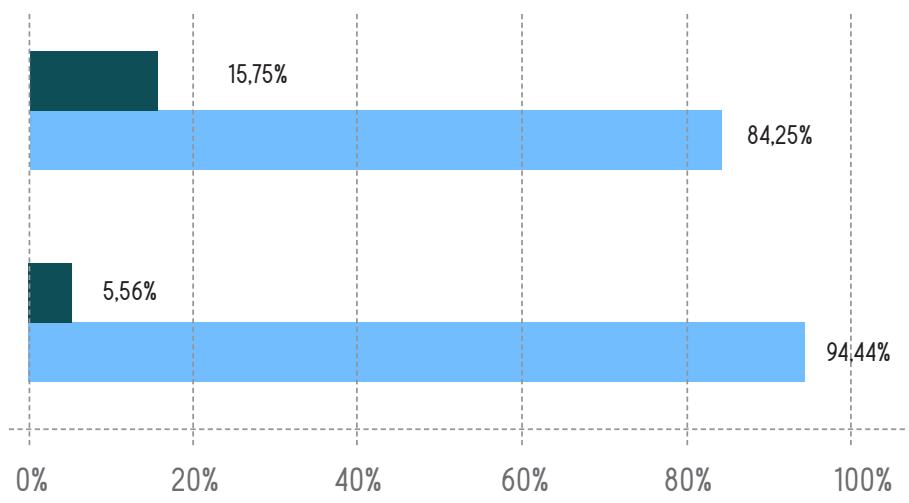

SEMANA 1 (DEL 7 AL 13) 


\subsection{Reacciones sobre el muro de pago}

En cuanto a las reacciones que este asunto suscitó en Twitter, la mayoría de los mensajes registrados sobre el muro de pago son de carácter negativo (49 de los 53, lo que supone un $92,45 \%)$, solo contabilizándose 4 tuits (7,55\%) en los que el usuario transmite una opinión favorable a la aplicación del límite de visitas.

Respecto a los mensajes de rechazo al nuevo modelo de negocio implantado por Sur, en los tuits registrados se critican, principalmente, la aplicación del muro de pago 0 el propio modelo como concepto o medida $(26,41 \%)$, la calidad periodística de los contenidos del medio $(11,32 \%)$ y la excesiva publicidad con la que cuenta la web del medio $(9,43 \%)$.

"Menuda idea. Ya no se pueden leer muchas de las noticias si no te suscribes, con la publicidad que metéis ya es suficiente" (publicado por @kikOliva, 14-05-2019) es uno de los ejemplos de mensajes críticos con la publicidad existente en la edición digital, al igual que el publicado por el usuario @DiegoJDP99 en el que indica: “Quitad esto porque ya ganáis suficiente dinero con los anuncios como para poneros a vender noticias sobre mi ciudad ahora. Esto es ya el colmo, ni que fuerais Netflix, que solo os ven gente de Málaga" (16-05-2019). Algunos ejemplos de tuits en los que se cuestiona la calidad de los contenidos del medio son: "Para que las noticias sean veraces y contrastadas y les deis oportunidad a la otra parte de hablar tenéis que hacer otro periódico, con gente nueva que no les dé miedo de decir las noticias tal como son" (@JUANAGARCADAZ1, 12-05-2019) 0 “Información ‘contrastada’ y ‘veraz' dice... [Acompañado de varios emoticonos de risa]” (@ DarthKssini, 12-05-2019), entre otros.

En cuanto a los mensajes de rechazo al formato o el modelo en sí, diversos usuarios se manifiestan de la siguiente manera: "La idea de cobrar por esto es de bombero" (@MiguelSalgadoH, 14-05-2019), “En breve habrá un ERE... Disminuyen los lectores, las empresas pondrán publicidad en la competencia, menos ingresos, más despidos... Sois unos cracks" (@littleSophieMLG, 15-05-2019). Otro usuario, por su parte, sugiere en tono sarcástico: “¡Vale! ¿Me podéis resetear la cuenta desde hoy? Y así me guardo una noticia para las elecciones, otra para el ascenso del Málaga C.F. y otra para un bonus por si ocurre algo imprevisible. Gracias. 5 noticias al mes es de coña” (@costann, 16-05-2019).

Todas estas circunstancias y motivos de crítica hacen que el $43,4 \%$ de los usuarios que interacciona con el medio en dicho periodo y aborda esta cuestión afirme que dejará de leer la cabecera de Vocento, optando, a partir de la aplicación del cobro por contenidos, por recurrir a otros medios locales, como Málaga Hoy y La Opinión de Málaga, 
para estar informado. Respecto a esta tipología de tuits, en el 37,73\% (20 mensajes) de ellos se manifiesta esta intención sin argumentar cuál es el motivo (calidad, precio, publicidad, etc.) por el que dejarán de leer Sur y no se suscribirán. Tan solo en el 5,67\% se indica de manera detallada la razón.

Frente a esto, en una notable menor medida los lectores se muestran favorables al límite de visitas gratuitas, registrándose, como se ha comentado anteriormente, un total de 4 tuits de carácter positivo. Asimismo, en tres de ellos $(5,66 \%)$ los usuarios indican que se suscribirán a SUR ON+.

\section{FIGURA 2}

\section{Contenido de los tuits referidos al muro de pago}

\begin{tabular}{|c|c|c|c|}
\hline Contenido del tuit & Semana 1 & Semana 2 & Total \\
\hline Manifiesta que dejará de leer el medio & 1 & 19 & $20(37,73 \%)$ \\
\hline Critica por la aplicación del muro de pago & 3 & 11 & $14(26,41 \%)$ \\
\hline Critica y cuestiona la calidad del medio & 4 & 2 & $6(11,32 \%)$ \\
\hline Critica la excesiva presencia de publicidad & 1 & 4 & $5(9,43 \%)$ \\
\hline Indica que se suscribirá a SUR ON+ & 1 & 2 & $3(5,66 \%)$ \\
\hline Critica por la publicidad y el precio de la suscripción & 1 & - & $1(1,89 \%)$ \\
\hline $\begin{array}{l}\text { Manifiesta que dejará de ser lector de Sur y critica el precio } \\
\text { de la suscripción }\end{array}$ & 1 & - & $1(1,89 \%)$ \\
\hline $\begin{array}{l}\text { Manifiesta que dejará de ser lector de Sur y critica la } \\
\text { excesiva presencia de publicidad en la web }\end{array}$ & - & 1 & $1(1,89 \%)$ \\
\hline $\begin{array}{l}\text { Manifiesta que dejará de ser lector de Sur y cuestiona la } \\
\text { calidad periodistica del medio }\end{array}$ & - & 1 & $1(1,89 \%)$ \\
\hline El usuario se muestra favorable a esta medida & 1 & - & $1(1,89 \%)$ \\
\hline TOTAL & 13 & 40 & $53(100 \%)$ \\
\hline
\end{tabular}




\section{Conclusiones}

Como se ha visto, más allá del motivo principal de rechazo a SUR ON+, los lectores de la cabecera de Vocento se muestran notablemente críticos en Twitter ante el anuncio de un nuevo modelo de negocio basado en el cobro por el acceso a los contenidos. Especialmente a partir del 14 de mayo, fecha en la que se comenzó a aplicar esta medida, las voces críticas contra Sur se multiplicaron, al considerar muchos usuarios que este medio les estaba privando del acceso a la información. No obstante, a pesar de esta mayor actividad o interacción entre los días 14 y 20 de mayo en comparación a los días previos a la puesta en marcha del modelo objeto de estudio, en ninguna de las dos semanas la incidencia de este asunto respecto al total de tuits dirigidos a la cuenta del medio alcanza valores significativos, por lo que su repercusión o alcance en Twitter es moderado. Con ello queda respondido el 01.

El 02 pretendía conocer cuáles han sido las cuestiones más destacadas que se han abordado en aquellos tuits relativos al muro de pago, así como si estos mensajes eran, en su mayoría, de carácter positivos o negativos. En este sentido, como se ha comentado, la casi totalidad de los mensajes son negativos o de rechazo a SUR ON+. Unas críticas basadas, principalmente, en el hecho de tener que pagar por la información, la presencia de publicidad en la edición digital del diario y la cuestionada calidad de sus contenidos periodísticos. Sin embargo, estas quejas de los internautas afloran una vez anunciada esta medida, existiendo, además, un núcleo duro de tuiteros que a lo largo de los días reiteran su discurso y publican varios mensajes contrarios al muro de pago.

Por último, vinculado con la afirmación anterior y como se plantaba en el 03, aproximadamente en la mitad de los tuits (49,06\%) los usuarios manifiestan cuál será su postura, en relación a su continuidad como lectores del medio, una vez que se inicie el cobro por el acceso a los contenidos. Mayoritariamente admiten que dejarán de leer las publicaciones de Sur, solo registrándose una cifra muy reducida de tuits en los que los usuarios transmiten una opinión favorable sobre esta medida. Esta poca incidencia -tanto de la temática en general como de las opiniones favorables hacia él- puede explicarse dado que, si bien Twitter es una red social en la que las personas publican tanto sus opiniones a favor como contrarias sobre algo o alguien, son, precisamente, los mensajes negativos y/o las críticas las que centran predominantemente la atención de los usuarios que escriben en esta red. Así, suele haber mayor actividad para realizar una queja que para destacar otras cuestiones neutrales o positivas -en este caso, el muro de pago y la posibilidad de suscribirse-. Por tanto, aquellos lectores cuya voluntad es seguir informándose a través de 
la cabecera de Vocento no se hacen presente de la misma manera en Twitter que los que muestran su rechazo.

Esto último podría llamar a posibles investigaciones o estudios en los que se aborden la evolución del número de lectores que ha experimentado Sur desde la puesta en marcha de su modelo de pago. También resultaría interesante realizar un análisis diacrónico en el que se determine si el denominado núcleo duro que anteriormente señalábamos continúa a lo largo del tiempo publicando mensajes críticos con el muro de pago, así como un estudio exploratorio en el que se analice si, pasado un tiempo, los usuarios que manifestaron su voluntad de no volver a leer este medio comentaron o compartieron alguna publicación de la cabecera objeto de estudio. No se nos ocultan las limitaciones de la investigación, pues el análisis se ha ceñido solo a las impresiones relativas al muro de pago de SUR ON+. Aun así, los hallazgos permiten dilucidar la escasa aceptación que, a priori, tiene esta medida entre los lectores de prensa que se manifiestan en Twitter.

Este artículo invita a la reflexión sobre los nuevos modelos de negocio que están adoptando las empresas informativas, así como en la relación y comunicación de estas con sus audiencias, prestando atención a las quejas o reclamos de los lectores con el propósito de mantenerlos -y aumentar la audiencia- una vez que el medio comienza a poner en marcha medidas como el límite de visitas gratuitas o la suscripción para poder acceder a la edición digital. Las reacciones recogidas demuestran, además, de manera parcial, dos hechos. Por una parte, que la cultura o filosofía de "todo gratis" está fuertemente arraigada en la sociedad y, por otra, que la información y la profesión periodística están devaluadas al punto de que la ciudadanía no se muestra favorable a pagar por los contenidos periodísticos.

\section{Bibliografía}

Aguado, J.M. \& Castellet, A. (2013). Periodismo móvil e información ubicua. En J.M. Aguado, C. Feijóo e I.J. Martínez (coords.), La comunicación móvil. Hacia un nuevo ecosistema digita. Barcelona: Gedisa, 187-218.

Asociación de Medios de Información (AMI) (2017). El Libro Blanco de la Información 2017. Madrid: AMI. 
Barredo, D. (2015). El análisis de contenido. Una introducción a la cuantificación de la realidad. Revista San Gregorio, (1), 26-31. Recuperado de http://revista. sangregorio.edu.ec/index.php/REVISTASANGREGORI0/article/view/113/67

Campos-Freire, F.; Rúas-Araújo, J.; López-García, X. \& Martínez-Fernández, V.A. (2016). Impacto de las redes sociales en el periodismo. El profesional de la información, 25(3), 449-457. D0I: http://dx.doi.org/10.3145/epi.2016.may.15

Campos-Freire, F.; Yaguache, J. \& Ulloa, N. (2017). Tendencias de la industria de los medios de América del Sur en la transición digital. Revista de Comunicación, 16(2), 33-56. D0I: https://doi.org/10.26441/RC16.2-2017-A2

De Aguilera, M. \& Casero-Ripollés, A. (2018). ¿Tecnologías para la transformación? Los medios sociales ante el cambio político social. Icono 14, 16(1), 1-21. D0I: http://dx.doi.org/10.7195/ri14.v16i1.1162

García Avilés, J.A. \& Arias Robles, F. (2016). Géneros periodísticos en los formatos visuales de Twitter: una propuesta de tipología. Textual y Visual Media, (9), 101 132. Recuperado de https://dialnet.unirioja.es/servlet/articulo? codigo $=6502733$

García Galindo, J.A. (2010). La opinión pública en la sociedad de la información: un fenómeno social en permanente cambio. BROCAR, (34), 273-288. DOI: http:// dx.doi.org/10.18172/brocar.1653

Gómez-Borrero, P. (2014). Estrategias de pago en diarios digitales en el mundo. Instituto para la Innovación Periodística. Recuperado de http://www.2ip.es/ estrategias-de-pago-en-diarios-digitales-en-el-mundo/

González Alba, J.A. (2018). ¿Qué papel juegan Google y Facebook en el futuro del periodismo? Cuadernos de Periodistas, (35), 56-70. Recuperado de http://www. cuadernosdeperiodistas.com/media/2018/04/Jose_Antonio_Gonzalez.pdf

Guallar, J. (2015). Prensa digital en 2013-2014. Anuario ThinkEPI, (9), 153-160. DOI: http://dx.doi.org/10.3145/thinkepi.2015.37

Igartua Perosanz, J.J. (2006). Métodos cuantitativos de investigación en comunicación. Barcelona: Bosch. 


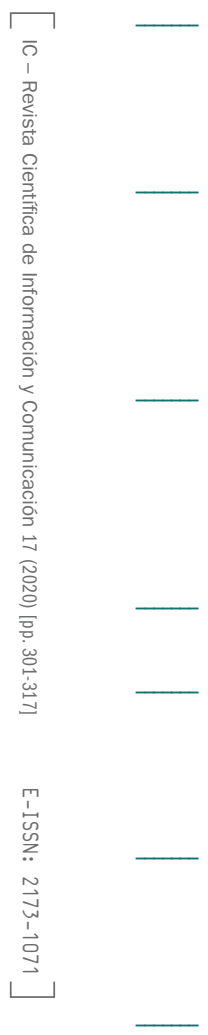

Infoadex (2019). La inversión publicitaria disminuye un -2,2\% el primer semestre de 2019. Recuperado de https://www.infoadex.es/home/la-inversion-publicitariadisminuye-un-22-el-primer-semestre-de-2019/

Justel-Vázquez, S.; Fernández-Planells, A.; Victoria-Mas, M. \& Lacasa-Mas, I. (2018). Twitter e información política en la prensa digital: la red social como fuente de declaraciones en la era Trump. El profesional de la información, 27(5), 984992. DOI: https://doi.org/10.3145/epi.2018.sep.03

Meso, K.; Larrondo, A.; Peña, S. \& Rivero, D. (2014). Audiencias activas en el ecosistema móvil: Análisis de las opciones de interacción de los usuarios en los cibermedios españoles a través de la web, los teléfonos móviles y las tabletas. Hipertext.net, (12). DOI: https://doi.org/10.2436/20.8050.01.9

Neuendorf, K.A. (2002). The Content Analysis Guidebook. Thousand Oaks: Sage.

Ormaetxea, M. (2014). Los muros de pagos no salvarán a la prensa. Cuadernos de Periodistas, (29), 55-73. Recuperado de http://www.cuadernosdeperiodistas. com/media/2015/04/ORMAETXEA.pdf

Palau-Sampio, D. (2016). Metamorfosis de la prensa de referencia en el contexto digital: clickbait y estrategias de tabloide en Elpais.com. Communication \& Society, 29(2), 63-80. Recuperado de http://dadun.unav.edu/handle/10171/41898

Palomo, B. \& Palau-Sampio, D. (2016). El periodista adaptativo. Consultores y directores de innovación analizan las cualidades del profesional de la comunicación. El profesional de la información, 25(2), 188-195. DOI: http:// dx.doi.org/10.3145/epi.2016.mar.05

Peñafiel, C. (2016). Reinvención del periodismo en el ecosistema digital y narrativas transmedia. AdComunica, (12), 163-182. DOI: http://dx.doi.org/10.6035/21740992.2016 .12

Picard, R.G. (2014). Twilight or new dawn of journalism. Evidence from the changing news ecosystem. Journalism studies, 15(5), 500-510. D0I: https:// doi. org/10.1080/1461670X.2014.895530 
Rodríguez, E. F. \& Favaretto, C. (2017). ¿Es rentable el periodismo de calidad? Disposición al pago en Brasil y España. TransInformaçao, 29(2), 211-220. DOI: https://doi.org/10.1590/2318-08892017000200008

Salaverría, R. (Coord.). (2005). Cibermedios. El impacto de internet en los medios de comunicación en España. Sevilla: Comunicación Social Ediciones y Publicaciones.

Wimmer, R.D. \& Dominick, J.R. (1996). La investigación científica de los medios de comunicación. Una introducción a sus métodos. Barcelona: Bosch. 
